\title{
Novel Oxytocin Receptor Variants in Laboring Women Requiring High Doses of Oxytocin
}

\author{
Erin L. Reinl, PhD ${ }^{1,{ }^{*}}$, Mr. Zane A. Goodwin ${ }^{2,{ }^{*}}$, Nandini Raghuraman, MD ${ }^{1}$, Ms. Grace Y. Lee ${ }^{1}$, \\ Ms. Erin Y. Jo ${ }^{1}$, Ms. Beakal M. Gezahegn ${ }^{1}$, Ms. Meghan K. Pillai ${ }^{1}$, Alison G. Cahill, MD ${ }^{3}$, \\ Cristina De Guzman Strong, $\mathrm{PhD}^{2}$, and Sarah K. England, $\mathrm{PhD}^{1}$ \\ ${ }^{1}$ Department of Obstetrics and Gynecology, Center for Reproductive Health Sciences, \\ Washington University in St. Louis, School of Medicine, St. Louis, MO \\ 2Division of Dermatology, Center for Pharmacogenomics, Center for the Study of Itch, \\ Department of Medicine, Washington University in St. Louis, School of Medicine, St. Louis, MO \\ ${ }^{3}$ Department of Obstetrics and Gynecology, Washington University in St. Louis, School of \\ Medicine, St. Louis, MO
}

\begin{abstract}
Background-Although oxytocin is commonly used to augment or induce labor, it is difficult to predict its effectiveness because oxytocin dose requirements vary significantly amongst women. One possibility is that women requiring high or low doses of oxytocin have variations in the oxytocin receptor gene.
\end{abstract}

Objectives-This work aims to identify oxytocin receptor gene variants in laboring women with low and high oxytocin dosage requirements.

Study Design-Term, nulliparous women requiring oxytocin doses of $\leq 4$ milliunits/minute (low-dose requiring, $n=83$ ) or $\geq 20$ milliunits/minute (high-dose requiring, $n=104$ ) for labor augmentation or induction were consented to a post-partum blood draw as a source of genomic DNA. Targeted-amplicon sequencing (coverage > 30X) with Illumina MiSeq was performed to discover variants in the coding exons of the oxytocin receptor gene. Baseline relevant clinical history, outcomes, demographics, and oxytocin receptor gene sequence variants and their allele frequencies were compared between low-dose-requiring and high-dose-requiring women. The Scale-Invariant Feature Transform algorithm was used to predict the effect of variants on oxytocin receptor function. Fisher's exact or chi-squared tests were used for categorical variables, and

Corresponding Author: Sarah K. England, 425 S. Euclid Ave., St. Louis, MO 63110, Ph. (314) 747-0937, Cell: (319) 621-4628, Fax: (314) 747-0264.

Co-first authors.

Publisher's Disclaimer: This is a PDF file of an unedited manuscript that has been accepted for publication. As a service to our customers we are providing this early version of the manuscript. The manuscript will undergo copyediting, typesetting, and review of the resulting proof before it is published in its final citable form. Please note that during the production process errors may be discovered which could affect the content, and all legal disclaimers that apply to the journal pertain.

Disclosures: The authors report no conflicts of interest.

Findings were presented, in part, at the $63^{\text {rd }}$ annual Society for Reproductive Investigation meeting in Montreal, Canada, March 16 19, 2016. 
Student $t$-tests or Wilcoxon rank sum tests were used for continuous variables. A $P$-value $<0.05$ was considered statistically significant.

Results-The high-dose-requiring women had higher rates of obesity and diabetes and were more likely to have undergone labor induction and required prostaglandins. High-dose-requiring women were more likely to undergo cesarean for first stage arrest and less likely to undergo cesarean for non-reassuring fetal status. Targeted sequencing of the oxytocin receptor gene in the total cohort ( $\mathrm{n}=187)$ revealed 30 distinct coding variants: 17 non-synonymous, 11 synonymous, and two small structural variations. One novel variant (A243T) was found in both the low- and high-dose-requiring groups. Three novel variants (Y106H, A240_A249del, and P197delfs*206) resulting in an amino acid substitution, loss of 9 amino acids, and a frameshift stop mutation, respectively, were identified only in low-dose-requiring women. Nine non-synonymous variants were unique to the high-dose-requiring group. These included three known variants (R151C, G221S, and W228C) and six novel variants not found in Ensembl or ExAC (M133V, R150L, H173R, A248V, G253R, and I266V). Of these, R150L, R151C, and H173R were predicted to damage oxytocin receptor function. There was no statistically significant association between the numbers of synonymous and non-synonymous substitutions in the patient groups.

Conclusions-Obesity, diabetes, and labor induction were associated with the requirement for high doses of oxytocin. We did not identify significant differences in the prevalence of oxytocin receptor variants between low-dose-requiring and high-dose-requiring women, but novel oxytocin receptor variants were enriched in the high-dose-requiring women. Additionally, we found three oxytocin receptor variants (two novel, one known) that were predicted to damage oxytocin receptor function and would likely increase an individual's risk for requiring a high oxytocin dose. Further investigation of oxytocin receptor variants and their effects on protein function will inform precision medicine in pregnant women.

\section{Keywords}

Induction of labor; labor augmentation; oxytocin; oxytocin receptor; pregnancy

\section{Introduction}

Oxytocin is commonly used to induce or augment labor and is one of the most frequently used medications in obstetrics. This nonapeptide hormone, which is secreted by the pituitary gland, corpus luteum, placenta, amnion, and decidua, acts on uterine smooth muscle to initiate, enhance, and pace uterine contractions ${ }^{1-3}$. On average, nearly half of women who give birth in the United States are administered synthetic oxytocin to induce or augment labor ${ }^{4,5}$. However, the effectiveness of a given oxytocin dose is variable among women; consequently, oxytocin has a relatively wide therapeutic window between $1 \mathrm{mU} / \mathrm{min}$ and higher than $40 \mathrm{mU} / \mathrm{min}$.

Frey and colleagues recently identified several risk factors for requiring high doses of oxytocin, including diabetes, intrapartum fever, and large fetal birthweight ${ }^{6}$. However, many women with these risk factors only require low doses of oxytocin, and those without any risk factors may require high doses of oxytocin, indicating an incomplete understanding of the etiologies of high oxytocin dose requirement. The unpredictability of an individual patient's 
sensitivity to exogenous oxytocin and subsequent prolonged oxytocin exposure increases the risks of uterine rupture, hemorrhage, hyponatremia, tachysystole, and fetal hypoxia, acidemia, and abnormal heart rate tracings ${ }^{7}$. Furthermore, the variability of responsiveness to oxytocin may contribute to the rising national cesarean delivery rate for the indication of labor dystocia.

Oxytocin induces uterine contractility by binding to the oxytocin receptor (OXTR), a Gprotein coupled receptor. Binding initiates a signaling cascade that results in an increased intracellular calcium concentration and activation of the contractile machinery. To successfully initiate the signaling cascade, OXTR must 1) properly bind oxytocin, 2) interact with its G-protein and/or other associated signaling molecules, and 3) translate oxytocin binding to a signal, or conformational change, that activates the G-protein. Protein coding variants of OXTR that enhance or interfere with these properties could lead to increased or decreased oxytocin sensitivity.

$O X T R$ variants have been associated with neurologic disorders including autism spectrum disorders and schizophrenia ${ }^{8-12}$. However, many variants identified in these studies were in non-coding regions surrounding the gene, and effects on function are unknown. Kim et al. (2013) investigated the link between $O X T R$ variants and preterm birth ${ }^{29}$ but did not find any variants that were statistically associated with preterm birth. The objective of our study was to identify $O X T R$ variants in women requiring low or high doses of oxytocin during labor induction or augmentation.

\section{Materials and Methods}

\section{Study Cohort and Inclusion and Exclusion Criteria}

This prospective study included nulliparous women at or beyond 37 weeks of gestation with singleton, non-anomalous pregnancies who received oxytocin for labor or induction of labor at a tertiary care medical center, between September 2013 and May 2016. Institutional review board approval was obtained from the Washington University School of Medicine Human Research Protection Office (IRB 201108143, approved 9/22/11). Exclusion criteria included a history of HIV or hepatitis, multiple pregnancy, ages $<13$ or $>48$ years old, or received magnesium. Patients meeting inclusion criteria were enrolled and consented postpartum by trained research nurses.

\section{Group Assignments}

The low-dose-requiring (LDR) women were defined as those who received a maximum dose of $\leqslant 4 \mathrm{mU} / \mathrm{min}$ oxytocin, and high-dose-requiring (HDR) women were those who received $\geq 20 \mathrm{mU} / \mathrm{min}$ oxytocin. These groups were selected because they fell below the $25^{\text {th }}$ percentile and above the $95^{\text {th }}$ percentile of oxytocin dose within our population of laboring patients ${ }^{6}$. Our institutional protocol for oxytocin administration allows for either high-dose or low-dose regimens at the discretion of the obstetric provider. The low-dose regimen starts at an initial rate $2 \mathrm{mU} / \mathrm{min}$ with an increase of $2 \mathrm{mU} / \mathrm{min}$ every 20 minutes to a maximum of $40 \mathrm{mU} / \mathrm{min}$. The high-dose regimen starts at $6 \mathrm{mU} / \mathrm{min}$ with an increase of $6 \mathrm{mU} / \mathrm{min}$ every 20 minutes to a maximum of $40 \mathrm{mU} / \mathrm{min}$. The LDR group exclusively included women on 
the low-dose regimen, and the HDR group included women on either the high- or low-dose regimens. Trained research nurses reviewed medical charts to extract pertinent clinical history, outcomes, and demographics.

\section{DNA Extraction}

Consenting women underwent a post-partum blood draw, and genomic DNA was extracted from blood samples by a salting-out procedure described by Miller et al., or with QIAamp DNA Blood Mini and Midi Kits (Qiagen, Germantown, MD) ${ }^{13}$. DNA concentrations and initial quality ratio of absorbance at $260 \mathrm{~nm}$ to absorbance at $230 \mathrm{~nm}>1.8$ ) were assessed by Nanodrop 1000 Thermo Scientific, Waltham, MA).

\section{Illumina MiSeq Sequencing}

PCR amplicons that tiled across the OXTR coding region were designed with the Fluidigm Assay Design Services. The sample inlets consisted of 1X High Fidelity FastStart Reaction Buffer without $\mathrm{MgCl}_{2}$ (Roche), $4.5 \mathrm{nM} \mathrm{MgCl} 2$ (Roche), 5\% DMSO (Roche), $200 \mu \mathrm{M}$ PCR Grade Nucleotide Mix (Roche), 0.05 U/ $\mu$ L FastStart High Fidelity Enzyme Blend (Roche), 1X Access Array Loading Reagent (Fluidigm), 75 ng DNA, and water. Forward and reverse primers (Table S1) were added to the assay inlets at $200 \mathrm{nM}$ each in $1 \mathrm{X}$ Access Array Loading Reagent. Multiplex PCR amplification was performed on the Fluidigm BioMark HD system with each patient sample, which was also tagged with a 10 base pair index. Samples were pooled and bead-purified before sequencing (MiSeq, 250 bp paired-end read length).

\section{Variant Identification and Filtering}

Sequence data were analyzed with a custom pipeline. Raw sequence reads were trimmed to remove primer sequences and aligned to human reference sequence hg19 by using Novoalign v2.08.02. Variants were called with FreeBayes v0.9. $7^{14}$ and then filtered with SnpSift for quality scores of 1000 or greater, depth of 40 or more reads, and percent of paired reads $\geq 20 \%$. Variants were annotated with SnpEff ${ }^{15,16}$. Each variant call was supported by an average of 16,800 reads, and only reads with mapping quality $\geq 30$ were considered for analysis. For each variant identified in individual patients, base call quality was determined as the negative log-probability that a base had not been sequenced correctly. Mapping quality per identified variant was defined as the negative log-probability that a read mapped to a unique location in the $O X T R$ reference sequence. Variants were excluded from the study if $<10$ reads supported the alternate allele, $<20 \%$ of the reads supported the alternate allele, and if $<10$ reads mapped to a site in the $O X T R$ gene. Variants with a base call quality $<20$, a mapping quality $<30$, and a base call quality + mapping quality $<40$ were also excluded from the analysis. Variants were named according to the Human Genome Variation Society's guidelines for genetic and protein variant nomenclature ${ }^{17}$. SnpEff v3.3c was used to determine amino acid changes, and the Variant Effect Predictor was used to determine SIFT scores ${ }^{18}$. 


\section{Statistical Analyses}

Baseline characteristics were compared between LDR and HDR groups and were statistically assessed by using Fisher's exact or chi-squared tests for categorical variables and Student $t$-tests or Wilcoxon rank sum test for continuous variables. A $P$-value $<0.05$ was considered significant. Homozygous variants were assigned an allele count of 2 , and heterozygous variants were assigned an allele count of 1. Homozygous variants that matched the reference allele were assigned an allele count of 0 . Allele frequencies were calculated as the sum of alleles divided by the total number of possible alleles in the patient cohort (number of patients $\times 2$ ). Odds ratios, probabilities, and confidence intervals were calculated as described in Szumilas et al. with variant type (synonymous or non-synonymous) as the exposure variable ${ }^{19}$.

\section{Results}

Targeted sequencing of the OXTR gene was performed on DNA obtained from consented women who were treated with oxytocin for labor induction or augmentation (Figure 1). Of 187 women, 104 (56\%) were defined as HDR, and 83 (44\%) were LDR. One LDR sample failed to yield quality sequence data and was eliminated from any further analysis.

Compared to those in the LDR group, women in the HDR group were more likely to be obese, have diabetes, undergo induction of labor, and require prostaglandins for induction (Table 1). There was no difference in age, race, gestational age, or indication for induction between groups. After adjusting for confounders, HDR was associated with an increased risk of cesarean delivery for first stage arrest (aOR 5.4, 95\% CI 1.8-16.7) and a decreased risk of cesarean delivery for non-reassuring fetal status (aOR $0.3,95 \%$ CI $0.1-0.8$ ). There were no differences in rates of vaginal delivery, cesarean section, or completion of first stage of labor between the two groups (Table 2).

We identified 30 OXTR variants, of which17 were non-synonymous substitutions (NSS), 11 were synonymous substitutions (SS), and two were small structural variations (Table 3). Moreover, 10 variants were novel (i.e., not identified in Ensembl, ExAC, or the literature). ${ }^{20-22}$ Of these, eight were NSS (Y106H, R150L, M133V, H173R, A243T, A248V, G253R, and I266V), and two were deletions (A240_A249del, and P197delfs*206).

Because NSS variants were more likely to affect the structure and function of OXTR than SS variants, we sought to determine whether the HDR women had a higher burden of NSS OXTR variants than the LDR women. We identified 14 NSS and 6 SS variants in HDR women and 8 NSS and 7 SS in LDR women (Table 3 and data not shown). Twelve NSS variants were each identified in only one individual (either HDR or LDR) (minor allele frequency < 1\%) (Table 3). Relative risk assessment determined that HDR women were 1.31 $(0.78-2.44)$ times more likely than LDR women to have a NSS, but this was not statistically significant $(P=0.48)$. Both groups had a higher representation of SS variants than NSS variants (mean 59.6\% versus 33.2\%). A higher percentage of HDR than LDR women had NSS alleles ( $34.6 \%$ vs. $31.7 \%$ ), but this difference was not statistically significant $(P=0.68)$. These data show no significant difference between overall $O X T R$ variant load in oxytocin HDR vs. LDR women. 
To determine whether variants were likely to affect the structure and function of the OXTR protein, we used the SIFT algorithm, which classifies variants as "Damaging", "Tolerated", or "No Prediction" based on evolutionary conservation and the biochemical properties of the substituted amino acid ${ }^{23}$. SIFT classified 4 of the 17 non-synonymous $O X T R$ single nucleotide variants (SNVs) as "Damaging" (R150L, R151C, H173R, W195R), including three SNVs in the HDR group (R150L, R151C, H173R) (Table 3). Two of these, R150L and H173R, were novel. SIFT classified the remaining 13 SNVs as "Tolerated". We also identified two novel deletions in OXTR: a 27 bp deletion and a frameshift deletion (A240_A249del and P197Pdelfs*206) that are anticipated to result in a nine amino acid deletion and a frameshift stop-gain mutation, respectively.

\section{Comment}

Our study identified ten novel variants of $O X T R$, and six of these were unique to women requiring high doses of oxytocin. Both HDR and LDR women had more NSS variants than SS variants of $O X T R$, and HDR women had more potentially damaging variants. Although these findings were not statistically significant, they suggest a genetic component to oxytocin sensitivity in women undergoing labor induction or augmentation.

Four of the NSS variants were predicted by SIFT to be damaging (R150L, R151C, H173R, and W195R). A variant in R150, R150S, was previously identified in an individual with autism spectrum disorder ${ }^{24}$. This residue is part of the oxytocin receptor 'polar pocket', which changes conformation during receptor activation and is involved in G-protein interaction ${ }^{25-27}$. R151C was previously annotated in the Ensembl database (rs772841181), but its functional impact is not known. Given its polarity and proximity to R150, R151 may contribute to the stability of the polar pocket. H173 and W195 are located within the regions of the OXTR binding pocket that recognize the cyclic and linear, respectively, components of oxytocin ${ }^{25,28}$. The cyclic binding region is thought to confer more specificity for oxytocin binding than the region recognizing the linear portion of oxytocin, so the H173R and W195R mutations may differentially disrupt oxytocin binding strength or specificity. The other OXTR variants we identified in HDR women, G221S, W228C, V172A, L206V, A218T, and A238T, were all in the Ensembl and ExAC databases (see Table 3), but their phenotypic effects are unknown. Neither these nor the other nine $O X T R$ variants we found were predicted by SIFT to be damaging, but this does not preclude them from having unpredictable damaging effects on OXTR function. Future in vitro studies should reveal the effects of these variants on OXTR function.

Previously, Kim et al. (2013) investigated the link between $O X T R$ variants and preterm birth ${ }^{29}$ but did not find any variants that were statistically associated with preterm birth. Kim et al. identified some novel and damaging variants, including the more common variants in our study (V172A, L206V, A218T, and A238T), but we note no overlap in our studies between the extremely low-frequency (0.003) variants we found or the predicted damaging variants they found. By using a heterologous expression system, Kim et al. showed that some of their variants reduced oxytocin binding and $\mathrm{IP}_{3}$ production capability. Taken together, our studies suggest that oxytocin sensitivity is, at least in part, genetically determined. 
A strength of our study is our use of high-throughput targeted amplicon sequencing, which enabled us to discover and individually genotype the variants across the entire patient cohort. In contrast, several previous studies in search of genetic variation in disease and patient outcomes have relied on SNP microarrays and subsequent Sanger sequencing for validation. Another advantage of our study is that we were able to estimate the effects of the variants we identified by using software that has proven useful in predicting the molecular effects of variants in other genes ${ }^{30,31}$.

Our study has some potential limitations to consider. By only sequencing exons, we may have missed variants in the promoter affecting gene expression, enhancers affecting allelespecific expression, or splice acceptor site mutations in introns. Variants in these regions could affect overall $O X T R$ expression levels, which could also have a profound effect on oxytocin responsiveness, as myometrial OXTR levels rise in preparation for labor ${ }^{32,33}$. Additionally, the highly guanine rich region in the 5' end of prevented us from designing robust primers for PCR amplification and thus precluded our abilities to successfully sequence this region in any of the samples. We anticipate future high-throughput sequencing strategies to resolve the 5' end of $O X T R$ in women of interest, especially those with no other risk factors for decreased oxytocin sensitivity. Although we discovered 14 OXTR variants in HDR women, our study was not powered to investigate whether any of the variants contributed to LDR or HDR status. However, the results provide novel information about unique $O X T R$ variants that have not been previously identified or described.

This study corroborated the findings by Frey et al. demonstrating that maternal body mass index (BMI), maternal diabetes, and labor induction were risk factors for requiring high doses of oxytocin to achieve delivery ${ }^{6}$. We found that HDR patients were more likely than LDR patients to have cesarean sections for first stage arrest and less likely to have cesarean sections for non-reassuring fetal status. We suspect that those with non-reassuring fetal status were likely to proceed to cesarean section sooner, thereby limiting duration of oxytocin exposure and titration. There was no difference in second stage arrest between HDR and LDR women, suggesting that the genetic aspect of oxytocin sensitivity is most applicable for first stage management. The HDR women with NSS variants predicted to be damaging (H173R, R151C, and R150L) had the additional risk factor of labor induction. The subject with the R151C variant also had a high BMI $\left(35.7 \mathrm{~kg} / \mathrm{m}^{2}\right)$, and the subject with $\mathrm{R} 150 \mathrm{~L}$ had a high BMI $\left(45.5 \mathrm{~kg} / \mathrm{m}^{2}\right)$, pre-gestational diabetes, and large birthweight (4125 $\mathrm{g})$. Thus, although these women's requirements for a high dose of oxytocin cannot be attributed to the variants alone, the variants may add additional risk.

This study suggests that a genetic factor, variation in $O X T R$, is associated with oxytocin responsiveness during labor. Although these results are insufficient to make absolute conclusions about the effect of the OXTR variants we identified on uterine contractions and labor dystocia, they introduce novel genetic variants that will serve as a foundation for future work. Future studies should include a larger sample size of women to more fully assess the association between specific $O X T R$ variants, requirement for high and low oxytocin doses, and labor arrest diagnoses. Additionally, functional studies evaluating oxytocin binding, downstream signaling, and myometrial contractility should be performed to assess consequences of the variants we identified. In combination with our study, such experiments 
will improve our knowledge about genetic contributions to oxytocin responsiveness and may, in the long term, contribute to precision medicine in managing labor.

\section{Supplementary Material}

Refer to Web version on PubMed Central for supplementary material.

\section{Acknowledgments}

We would like to acknowledge Shannon Martin (Department of Obstetrics and Gynecology, Washington University in St. Louis, St. Louis, MO, in part funded by R21 HD076677-01, and March of Dimes grant 21-FY15-147 [to S.K.E., no conflicts of interest], 1F31HD079148-01 [to E.L.R, no conflicts of interest]), and the clinical research nurses for patient recruitment and data collection and Deborah Frank (Department of Obstetrics and Gynecology, Washington University in St. Louis, St. Louis, MO, no funding sources, no conflicts of interest) for critical review of this manuscript. We thank the Genome Technology Access Center in the Department of Genetics at Washington University School of Medicine for help with genomic analysis. The Center is partially supported by National Cancer Institute Cancer Center Support Grant \#P30 CA91842 to the Siteman Cancer Center and by Clinical and Translational Sciences Award Grant\# UL1 TR000448 from the National Center for Research Resources (NCRR), a component of the National Institutes of Health (NIH) and the NIH Roadmap for Medical Research. This publication is solely the responsibility of the authors and does not necessarily represent the official view of NCRR or NIH.

Financial Support: R21 HD076677-01 to S.K.E., F31 HD079148 to E.L.R., T32 HG000045 to Z.A.G, and R01AR065523 to CdGS.

\section{References}

1. Arrowsmith S, Wray S. Oxytocin: its mechanism of action and receptor signalling in the myometrium. J Neuroendocrinol. 2014; 26(6):356-369. [PubMed: 24888645]

2. Arthur P, Taggart MJ, Mitchell BF. Oxytocin and parturition: a role for increased myometrial calcium and calcium sensitization? Front Biosci. 2007; 12:619-633. [PubMed: 17127323]

3. Dale HH. The Action of Extracts of the Pituitary Body. Biochem J. 1909; 4(9):427-447. [PubMed: 16742120]

4. Osterman MJ, Martin JA, Menacker F. Expanded health data from the new birth certificate, 2006. Natl Vital Stat Rep. 2009; 58(5):1-24.

5. Martin JA, Hamilton BE, Ventura SJ, Osterman MJ, Wilson EC, Mathews TJ. Births: final data for 2010. Natl Vital Stat Rep. 2012; 61(1):1-72.

6. Frey HA, Tuuli MG, England SK, et al. Factors associated with higher oxytocin requirements in labor. J Matern Fetal Neonatal Med. 2015; 28(13):1614-1619. [PubMed: 25204333]

7. Simpson KR, Knox GE. Oxytocin as a high-alert medication: implications for perinatal patient safety. MCN Am J Matern Child Nurs. 2009; 34(1):8-15. quiz 16-17. [PubMed: 19104313]

8. Wermter AK, Kamp-Becker I, Hesse P, Schulte-Korne G, Strauch K, Remschmidt H. Evidence for the involvement of genetic variation in the oxytocin receptor gene (OXTR) in the etiology of autistic disorders on high-functioning level. Am J Med Genet B Neuropsychiatr Genet. 2010; 153B(2):629 639. [PubMed: 19777562]

9. Jacob S, Brune CW, Carter CS, Leventhal BL, Lord C, Cook EH Jr. Association of the oxytocin receptor gene (OXTR) in Caucasian children and adolescents with autism. Neurosci Lett. 2007; 417(1):6-9. [PubMed: 17383819]

10. Tost H, Kolachana B, Hakimi S, et al. A common allele in the oxytocin receptor gene (OXTR) impacts prosocial temperament and human hypothalamic-limbic structure and function. Proceedings of the National Academy of Sciences of the United States of America. 2010; 107(31): 13936-13941. [PubMed: 20647384]

11. Montag C, Brockmann EM, Bayerl M, Rujescu D, Muller DJ, Gallinat J. Oxytocin and oxytocin receptor gene polymorphisms and risk for schizophrenia: a case-control study. World J Biol Psychiatry. 2013; 14(7):500-508. [PubMed: 22651577] 
12. Souza RP, de Luca V, Meltzer HY, Lieberman JA, Kennedy JL. Schizophrenia severity and clozapine treatment outcome association with oxytocinergic genes. Int J Neuropsychopharmacol. 2010; 13(6):793-798. [PubMed: 20196918]

13. Miller SA, Dykes DD, Polesky HF. A simple salting out procedure for extracting DNA from human nucleated cells. Nucleic acids research. 1988; 16(3):1215. [PubMed: 3344216]

14. Garrison EMG. Haplotype-based variant detection from short-read sequencing. arXiv. 2012 preprint 2012(arXiv):1207.3907 [qbio.GN].

15. Cingolani P, Platts A, Wang leL, et al. A program for annotating and predicting the effects of single nucleotide polymorphisms, SnpEff: SNPs in the genome of Drosophila melanogaster strain w1118; iso-2; iso-3. Fly (Austin). 2012; 6(2):80-92. [PubMed: 22728672]

16. Cingolani P, Patel VM, Coon M, et al. Using Drosophila melanogaster as a Model for Genotoxic Chemical Mutational Studies with a New Program, SnpSift. Front Genet. 2012; 3:35. [PubMed: 22435069]

17. den Dunnen JT, Dalgleish R, Maglott DR, et al. HGVS Recommendations for the Description of Sequence Variants: 2016 Update. Hum Mutat. 2016; 37(6):564-569. [PubMed: 26931183]

18. McLaren W, Pritchard B, Rios D, Chen Y, Flicek P, Cunningham F. Deriving the consequences of genomic variants with the Ensembl API and SNP Effect Predictor. Bioinformatics. 2010; 26(16): 2069-2070. [PubMed: 20562413]

19. Szumilas M. Explaining odds ratios. J Can Acad Child Adolesc Psychiatry. 2010; 19(3):227-229. [PubMed: 20842279]

20. Sherry ST, Ward MH, Kholodov M, et al. dbSNP: the NCBI database of genetic variation. Nucleic acids research. 2001; 29(1):308-311. [PubMed: 11125122]

21. Yates A, Akanni W, Amode MR, et al. Ensembl 2016. Nucleic acids research. 2016; 44(D1):D710716. [PubMed: 26687719]

22. Lek M, Karczewski KJ, Minikel EV, et al. Analysis of protein-coding genetic variation in 60,706 humans. Nature. 2016; 536(7616):285-291. [PubMed: 27535533]

23. Ng PC, Henikoff S. Predicting deleterious amino acid substitutions. Genome Res. 2001; 11(5): 863-874. [PubMed: 11337480]

24. Liu X, Kawashima M, Miyagawa T, et al. Novel rare variations of the oxytocin receptor (OXTR) gene in autism spectrum disorder individuals. Hum Genome Var. 2015; 2:15024. [PubMed: 27081536]

25. Gimpl G, Fahrenholz F. The oxytocin receptor system: structure, function, and regulation. Physiol Rev. 2001; 81(2):629-683. [PubMed: 11274341]

26. Oliveira L, Paiva AC, Sander C, Vriend G. A common step for signal transduction in G proteincoupled receptors. Trends Pharmacol Sci. 1994; 15(6):170-172. [PubMed: 8091507]

27. Fanelli F, Barbier P, Zanchetta D, de Benedetti PG, Chini B. Activation mechanism of human oxytocin receptor: a combined study of experimental and computer-simulated mutagenesis. Mol Pharmacol. 1999; 56(1):214-225. [PubMed: 10385703]

28. Postina R, Kojro E, Fahrenholz F. Separate agonist and peptide antagonist binding sites of the oxytocin receptor defined by their transfer into the V2 vasopressin receptor. J Biol Chem. 1996; 271(49):31593-31601. [PubMed: 8940177]

29. Kim J, Stirling KJ, Cooper ME, et al. Sequence variants in oxytocin pathway genes and preterm birth: a candidate gene association study. BMC Med Genet. 2013; 14:77. [PubMed: 23889750]

30. Beecham AH, Patsopoulos NA, et al. International Multiple Sclerosis Genetics C. Analysis of immune-related loci identifies 48 new susceptibility variants for multiple sclerosis. Nat Genet. 2013; 45(11):1353-1360. [PubMed: 24076602]

31. Norton N, Li D, Rieder MJ, et al. Genome-wide studies of copy number variation and exome sequencing identify rare variants in BAG3 as a cause of dilated cardiomyopathy. Am J Hum Genet. 2011; 88(3):273-282. [PubMed: 21353195]

32. Fuchs AR, Fuchs F, Husslein P, Soloff MS. Oxytocin receptors in the human uterus during pregnancy and parturition. Am J Obstet Gynecol. 1984; 150(6):734-741. [PubMed: 6093538]

33. Soloff MS, Alexandrova M, Fernstrom MJ. Oxytocin receptors: triggers for parturition and lactation? Science. 1979; 204(4399):1313-1315. [PubMed: 221972] 
Samples collected: 194 (83 LDR, 111 HDR)

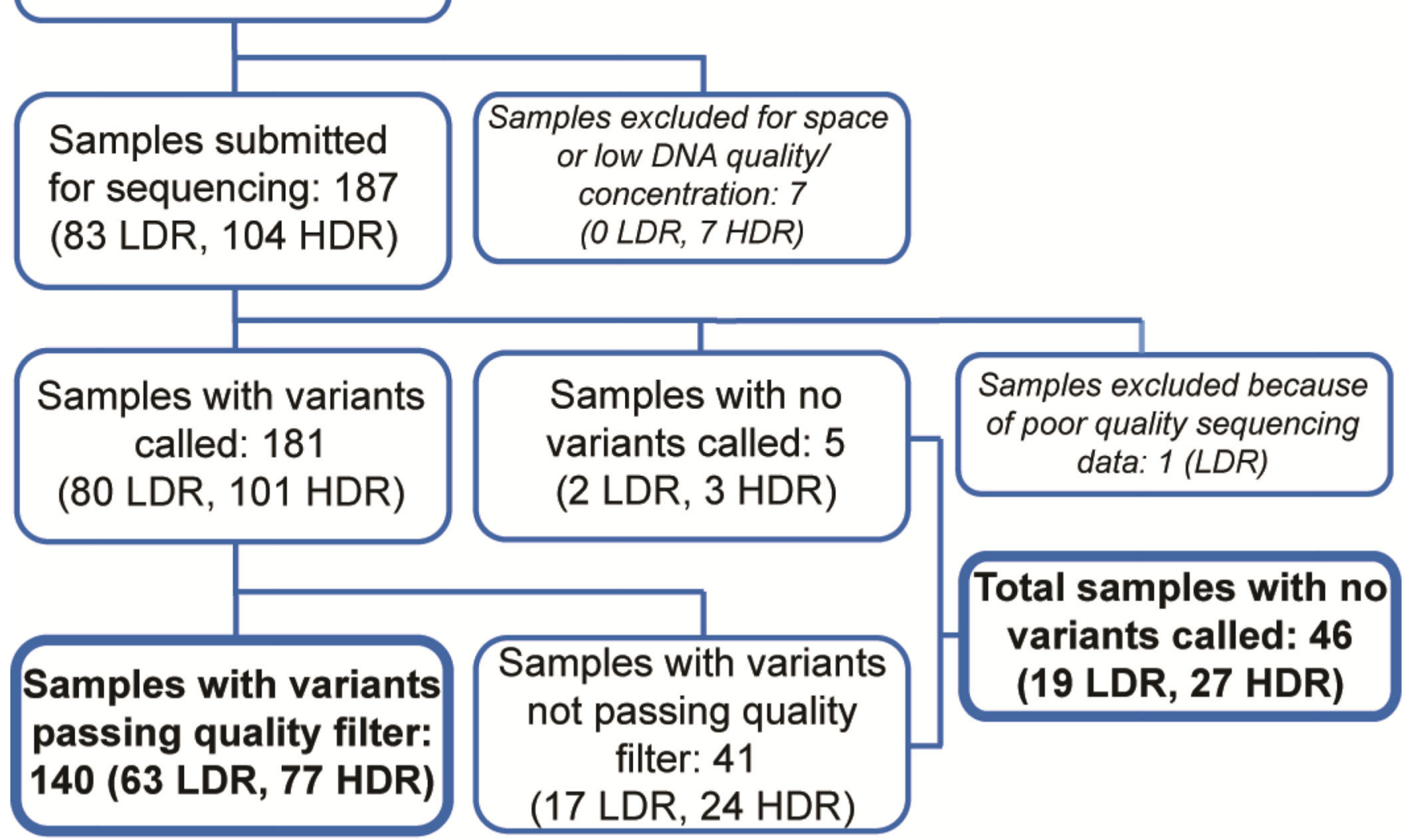

Figure 1.

Flow chart of sample and data collection. Samples included in final analysis are bolded. low dose requiring (LDR), high dose requiring (HDR). 


\section{Table 1}

Baseline demographics of cohort

\begin{tabular}{|c|c|c|c|}
\hline Variable & $\begin{array}{l}\text { LDR } \\
\mathrm{n}=82\end{array}$ & $\underset{n=104}{\text { HDR }}$ & $P$ \\
\hline Maternal Age (yrs) & $25 \pm 5.6$ & $26 \pm 6.0$ & 0.27 \\
\hline \multicolumn{4}{|l|}{ Race } \\
\hline African American & $49(59.8)$ & $54(51.9)$ & \multirow{4}{*}{0.50} \\
\hline Caucasian & $30(36.6)$ & $46(44.2)$ & \\
\hline Hispanic & $1(1.2)$ & $3(2.9)$ & \\
\hline Other & $2(2.4)$ & $1(1.0)$ & \\
\hline BMI $\left(\mathrm{kg} / \mathrm{m}^{2}\right)$ & $31.7 \pm 6.1$ & $35.0 \pm 7.4$ & $<0.01$ \\
\hline Obesity & $45(54.9)$ & $73(70.2)$ & 0.03 \\
\hline Gestational Age (days) & $273 \pm 19.6$ & $274 \pm 8.9$ & 0.54 \\
\hline Infant Birthweight (g) & $3245 \pm 482$ & $3319 \pm 540$ & 0.34 \\
\hline Smoking & $4(4.9)$ & $7(6.7)$ & 0.60 \\
\hline Illicit drug use & $7(8.5)$ & $6(5.8)$ & 0.46 \\
\hline Chronic hypertension & $2(2.4)$ & $5(4.8)$ & 0.40 \\
\hline Hypertensive disorder of pregnancy & $13(15.9)$ & $29(27.9)$ & 0.05 \\
\hline Diabetes & $0(0)$ & $10(9.6)$ & $<0.01$ \\
\hline Intrapartum Fever & $11(13.4)$ & $16(15.4)$ & 0.71 \\
\hline Epidural & $82(100)$ & $104(100)$ & -- \\
\hline Estimated blood loss (mL) & $548 \pm 322$ & $662 \pm 387$ & 0.03 \\
\hline Induction of labor & $38(46.3)$ & $81(77.9)$ & $<0.0001$ \\
\hline \multicolumn{4}{|l|}{ Indication for induction of labor } \\
\hline IUGR & $4(10.5)$ & $10(12.4)$ & 0.71 \\
\hline Hypertension & $13(34.2)$ & $24(29.6)$ & \\
\hline PROM & $2(5.3)$ & $11(13.6)$ & \\
\hline Fetal anomaly & $2(5.3)$ & $3(3.7)$ & \\
\hline Elective & $5(13.2)$ & $7(8.6)$ & \\
\hline Diabetes & $1(2.6)$ & $6(7.4)$ & \\
\hline Other & $11(29.0)$ & $20(24.7)$ & \\
\hline Prostaglandin & $25(30.5)$ & $47(45.2)$ & 0.04 \\
\hline
\end{tabular}

BMI, body mass index; LDR, low dose requiring; HDR, high dose requiring; IUGR, intrauterine growth restriction; PROM, premature rupture of membranes

Data represent mean \pm standard deviation or n (\%). P-value obtained by performing Student's t test, chi square test, or Fisher's exact test, as appropriate. 


\section{Table 2}

\section{Labor outcomes.}

\begin{tabular}{lcccc}
\hline Variable & $\begin{array}{c}\text { LDR } \\
\mathbf{n = 8 2}\end{array}$ & $\begin{array}{c}\text { HDR } \\
\mathbf{n = 1 0 4}\end{array}$ & $\mathbf{R R}(\mathbf{9 5 \%} \mathbf{C I})$ & aOR $^{*}(\mathbf{9 5 \%} \mathbf{C I})$ \\
\hline Cesarean Section & $32(39.0)$ & $53(51.0)$ & $1.3(0.94-1.82)$ & $1.4(0.7-2.6)$ \\
Indication for cesarean & & & & \\
First stage arrest & $4(4.9)$ & $27(26.0)$ & $\mathbf{5 . 3}(\mathbf{1 . 9}-\mathbf{1 4 . 6})$ & $\mathbf{5 . 4}(\mathbf{1 . 8}-\mathbf{1 6 . 7})$ \\
Second stage arrest & $5(6.1)$ & $9(8.7)$ & $1.4(0.5-4.1)$ & $1.9(0.6-6.5)$ \\
Non-reassuring fetal status & $15(18.3)$ & $8(7.7)$ & $\mathbf{0 . 4}(\mathbf{0 . 2}-\mathbf{0 . 9})$ & $\mathbf{0 . 3}(\mathbf{0 . 1}-\mathbf{0 . 8})$ \\
Other & $7(8.5)$ & $9(8.7)$ & $1.2(0.5-3.1)$ & $1.1(0.4-3.3)$ \\
Spontaneous Vaginal Delivery & $48(58.5)$ & $49(47.1)$ & $0.8(0.6-1.1)$ & $0.7(0.4-1.4)$ \\
Operative Vaginal Delivery & $2(2.4)$ & $2(1.9)$ & $0.8(0.1-5.5)$ & $0.9(0.1-7.4)$ \\
Completion of 1st stage & $55(67.1)$ & $63(60.6)$ & $0.9(0.7-1.1)$ & $1.0(0.5-1.9)$ \\
\hline
\end{tabular}

aOR, adjusted odds ratio; LDR, low dose requiring; HDR, high dose requiring; RR, relative risk

Adjusted for induction of labor and obesity

Data represents $\mathrm{n}(\%)$. RR calculated by chi square test. aOR estimated by multivariable logistic regression. 
